\title{
Intensive Insulin Protocol Improves Glucose Control and Is Associated with a Reduction in Intensive Care Unit Mortality
}

\author{
Charles C Reed, BSN, Ronald M Stewart, MD, FACS, Michele Sherman, BSN, John G Myers, MD, FACS, \\ Michael G Corneille, MD, FACS, Nanette Larson, BSN, Susan Gerhardt, MSN, Randall Beadle, BSN, \\ Conrado Gamboa, MS, RPh, Daniel Dent, MD, FACS, Stephen M Cohn, MD, FACS, \\ Basil A Pruitt Jr, MD, FACS
}

BACKGROUND: Intensive insulin therapy to maintain serum glucose levels between 80 and $110 \mathrm{mg} / \mathrm{dL}$ has previously been shown to reduce mortality in the critically ill; recent data, however, have called this benefit into question. In addition, maintaining uniform, tight glucose control is challenging and resource demanding. We hypothesized that, by use of a protocol, tight glucose control could be achieved in the surgical trauma intensive care unit (STICU), and that improved glucose control would be beneficial.

STUDY DESIGN: During the study period, a progressively more rigorous approach to glucose control was used, culminating in an implemented protocol in 2005. We reviewed STICU patients' blood glucose levels, measured by point-of-care testing, from 2003 to 2006. Mortality was tracked over the course of the study, and patient charts were retrospectively reviewed to measure illness and injury severity.

RESULTS: $\quad$ Mean blood glucose levels steadily improved $(\mathrm{p}<0.01)$. In addition to absolute improvements in glucose control, total variability of glucose ranges in the STICU steadily diminished. A reduction in STICU mortality was temporally associated with implementation of the protocol $(\mathrm{p}<0.01)$. There were fewer intraabdominal abscesses and fewer postinjury ventilator days after implementation of the protocol. There was a small increase in the incidence of clinically relevant hypoglycemia.

CONCLUSIONS: Improvements in glucose control in the ICU can be achieved by using a protocol for intensive insulin therapy. In our ICU, this was temporally associated with a significant reduction in mortality. (J Am Coll Surg 2007;204:1048-1055. (C) 2007 by the American College of Surgeons)

Insulin resistance and hyperglycemia are extremely common after surgical stress or injury, and have been associated with increased complications and death. ${ }^{1-5}$ The relationship of hyperglycemia to poor outcomes may be stronger in trauma patients than in other critically ill patients. ${ }^{6}$ Gore, Wu, Thomas, and colleagues ${ }^{7-9}$ from The University of

Competing Interests Declared: None.

This work was supported in part from a Trauma Institute of San Antonio grant.

Presented at the Southern Surgical Association 118th Annual Meeting, West Palm Beach, FL, December 2006.

Received December 3, 2006; Accepted December 15, 2006.

From the Surgical Trauma Intensive Care Unit, University Health System (Reed, Sherman, Corneille, Larson, Gerhardt, Beadle, Gamboa) and the Department of Surgery (Stewart, Myers, Dent, Cohn, Pruitt), University of Texas Health Science Center at San Antonio, San Antonio, TX.

Correspondence address: Ronald M Stewart, MD, Department of Surgery, University of Texas Health Science Center at San Antonio, 7703 Floyd Curl Dr, San Antonio, TX 78229.
Texas Medical Branch at Galveston (UTMB) have demonstrated improvements in protein synthesis and decreases in hepatic acute phase protein levels in severely burned and severely injured patients treated with intensive insulin therapy. In a group of primarily surgical patients, Van den Berghe and colleagues ${ }^{10}$ demonstrated significantly improved outcomes in patients randomized to receive intensive insulin therapy with target blood glucose levels in the range between $80 \mathrm{mg} / \mathrm{dL}$ and $110 \mathrm{mg} / \mathrm{dL}$. In 2006, the same group of authors reported on a similarly designed trial among medical intensive care patients. ${ }^{11}$ When analyzed along an intent-to-treat basis, there was no difference in mortality between the standard treatment group and the intensive insulin therapy group. But there were mortality benefits and reduced complications in the patients treated in the ICU longer than 3 days. Van den Berghe and colleagues ${ }^{11}$ noted that well-designed, randomized, clinical trials are needed to confirm their preliminary findings. 
We are aware of two large, prospective, randomized, multicenter trials designed to answer the question of whether intensive insulin therapy improves outcomes in critically ill patients: the Glucontrol and NICE-SUGAR trials. The Glucontrol trial has stopped enrollment, and preliminary findings have been presented at the European Society for Intensive Care Medicine. In contrast to Van den Berghe's results, there was no difference in survival between the routine care and the intensive insulin therapy groups, and there was an increased mortality in patients who developed hypoglycemia in the intensive insulin therapy arm of the trial. Maintaining strict glucose control is promising, but the technique is challenging and has not been uniformly adopted.

Following Van den Berghe and the group from the University of Texas Medical Branch, we hypothesized that by using a protocol, tight glucose control could be achieved in the surgical trauma intensive care unit (STICU), and that improved glucose control would be beneficial. We report on the process of implementing such a protocol and the results of this approach in a single surgical intensive care unit.

\section{METHODS}

\section{Study setting}

The study was performed in a single surgical and trauma intensive care unit at University Hospital, San Antonio, TX. University Hospital is a 604-bed, county taxsupported hospital. Physician services are provided by The University of Texas Health Science Center at San Antonio. The hospital functions as a tertiary referral center for a wide range of medical conditions and is the primary indigent care facility for Bexar County. The STICU is an "open" ICU with continuous in-hospital coverage by faculty intensivists and a multidisciplinary resident intensive care unit team.

As part of its tertiary care role, University Hospital, an American College of Surgeons verified Level I trauma center, serves as the lead trauma center for 22 counties in south central Texas. Its primary catchment area (Trauma Service Area P) encompasses a 26,904-square-mile region with 2.1 million residents. Its secondary catchment area includes Trauma Service Areas S, T, U, and V, extending from Laredo to Brownsville, and includes an additional 26,102 square miles and 2 million residents.

\section{Medical records data sources}

Mortality data were calculated by querying a prospectively maintained hospital information database for all admissions to the STICU from January 1, 2001 through October 31,2006 . Information on STICU deaths during the same period was obtained by querying a prospectively maintained hospital discharge and death database. Mortality rates were calculated by dividing the total number of deaths by the total number of patients admitted to the intensive care unit.

From 2003 to 2006, data on point-of-care testing of blood glucose levels and serum glucose levels were obtained by querying the hospital clinical information database. In years 2003, 2004, and 2005, charts were retrospectively abstracted for the 12 components of the APACHE II Acute Physiology Score, patient age, and the components of the APACHE II chronic health evaluation. In addition, the charts of all patients admitted to the STICU were abstracted for the preadmission diagnosis of diabetes, positive blood culture (two or more bottles), pneumonia (bronchoalveolar lavage of $>100,000$ organisms), diagnosis of intraabdominal abscess, gender, race/ethnicity, and admission and transfer times from the STICU.

The trauma registry was queried for diagnosis, Trauma and Injury Severity Score (TRISS), ventilator days, hospital days, ICU days, and discharge disposition. Registry software was used to calculate the probability of survival using TRISS methodology and the comparison to the multiinstitutional trauma outcomes study.

The data were analyzed by year and by two distinct time periods: 2003 to 2004 (period 1, before implementation of the protocol), and 2005 to 2006 (period 2, after implementation of the protocol).

\section{Protocol development}

Efforts to improve glucose control in the STICU began in 2002, after publication of the initial Van den Berghe and colleagues ${ }^{10}$ clinical trial. The process of improving glucose control developed along three distinct phases. The first phase consisted of physician assessment of the clinical evidence and a consensus that tight glucose control was feasible and important. During phase 1, the process of implementing intensive insulin therapy and tight glucose control was the responsibility of the faculty, residents, and nurses of the STICU.

The second phase began in 2004, after a perceived failure of the initial approach to achieve improved glucose control. Phase 2 began with the faculty, STICU nursing leadership, and the intensive care unit pharmacist meeting to develop a formal protocol for tight glucose control. The glucose control protocol was eventually implemented in the last quarter of 2004, one patient at a time. Feedback was used from these single patient trials to refine the protocol. The final protocol developed in phase 2 was fully implemented in January 2005.

Phase 3 began in July 2006, when it was perceived that the glucose protocol was effective in reducing hyperglyce- 
Table 1. Comparison of Patient Groups Before and After Initiation of Glucose Control Protocol

\begin{tabular}{lccc}
\hline Variable & Period 1 & Period 2 & p Value \\
\hline Age, $y$ & $48.5 \pm 0.4$ & $49.3 \pm 0.5$ & 0.28 \\
\hline Diabetes, \% & 19 & 19 & 0.48 \\
\hline TRISS Pd & $0.21 \pm 0.01$ & $0.19 \pm 0.01$ & 0.10 \\
\hline APACHE Pd & $0.22 \pm 0.004$ & $0.21 \pm 0.006$ & 0.20 \\
\hline Hospital, d & $14 \pm 0.5$ & $13 \pm 0.5$ & 0.26 \\
\hline ICU, d & $6.6 \pm 0.26$ & $6.0 \pm 0.27$ & 0.15 \\
\hline Ventilator, d & $3.1 \pm 0.3$ & $2.4 \pm 0.2$ & 0.03
\end{tabular}

Values are expressed as mean \pm SEM.

Pd, probability of death.

mia, but was not fully used in all hyperglycemic patients. This phase consisted of assigning a nurse to the designated role of quality improvement at the bedside. This nurse rounded on all patients in the STICU and performed a variety of quality improvement tasks, including ensuring that all hyperglycemic patients were enrolled into the glucose control protocol.

\section{Statistical analysis and predicted mortality assessment}

Control charting, Anova, chi-square, and Fisher's exact test were calculated using MedCalc for Windows, version 7.5 (MedCalc Software) and Microsoft Excel for Windows XP. Continuous variables are expressed as the mean \pm SEM.

APACHE II-predicted mortality rates were calculated for each patient by using the following formula: $\mathrm{Pd}=\mathrm{e}^{\mathrm{x}} /\left(1+\mathrm{e}^{\mathrm{x}}\right)$; $\mathrm{x}=-3.517+($ APACHE II score $\times 0.146)+$ DCW + ESW, where e is 2.718 (base of natural logarithm), Pd is the probability of death, DCW is diagnostic category weight, and ESW is emergency surgery weight. ${ }^{12}$ Each patient in the data set had a TRISS probability of survival calculated by the Digital Innovations Collector software and based on the model coefficients described by Boyd and associates. ${ }^{13}$ TRISS Probability of survival (Ps) was converted to Pd by the formula (1-Ps). The expected mortality of the group was calculated by computing the mean value of the individual probability of death for each patient. The observed minus expected mortality $\left(\mathrm{O}_{\mathrm{m}}-\mathrm{E}_{\mathrm{m}}\right)$ was calculated by taking the measured (observed) mortality and subtracting the calculated (expected) mortality using TRISS methodology (for trauma patients) or APACHE II methodology (for both trauma and nontrauma patients). As such, negative values for $\mathrm{O}_{m}-\mathrm{E}_{\mathrm{m}}$ represent a lower than expected mortality and positive values represent a higher than expected mortality.

\section{RESULTS}

Mortality data were calculated using 7,261 patients admitted from 2001 to 2006. Charts were abstracted on the 3,536 patients admitted to the STICU from January 1, 2003 to December 31, 2005. The mean age of the patients was 49 years. There were 1,252 women (35\%) and 2,284 men $(65 \%)$. Nineteen percent of the patients had a preadmission diagnosis of diabetes. Point-of-care blood glucose levels were available from 2003 to 2006 . Table 1 presents a comparison of the patients before and after implementation of the tight glucose control protocol. The mean blood glucose levels by year were: 2003, $141 \mathrm{mg} / \mathrm{dL} ; 2004,134$ $\mathrm{mg} / \mathrm{dL} ; 2005,129 \mathrm{mg} / \mathrm{dL}$; and 2006, $125 \mathrm{mg} / \mathrm{dL}$ (p < $0.01)$. A control plot of daily mean blood glucose values

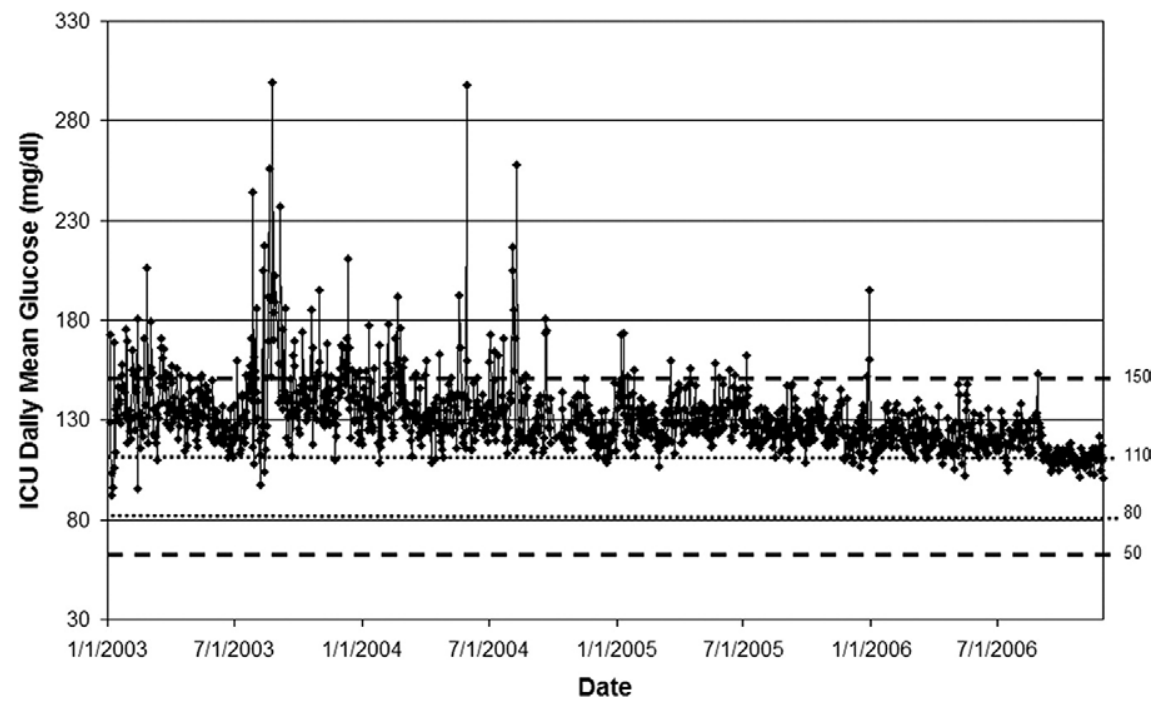

Figure 1. Running daily average of point-of-care blood glucose values from 2003 to 2006 . Mean blood glucose levels and variability of blood glucose values both decreased over time. 


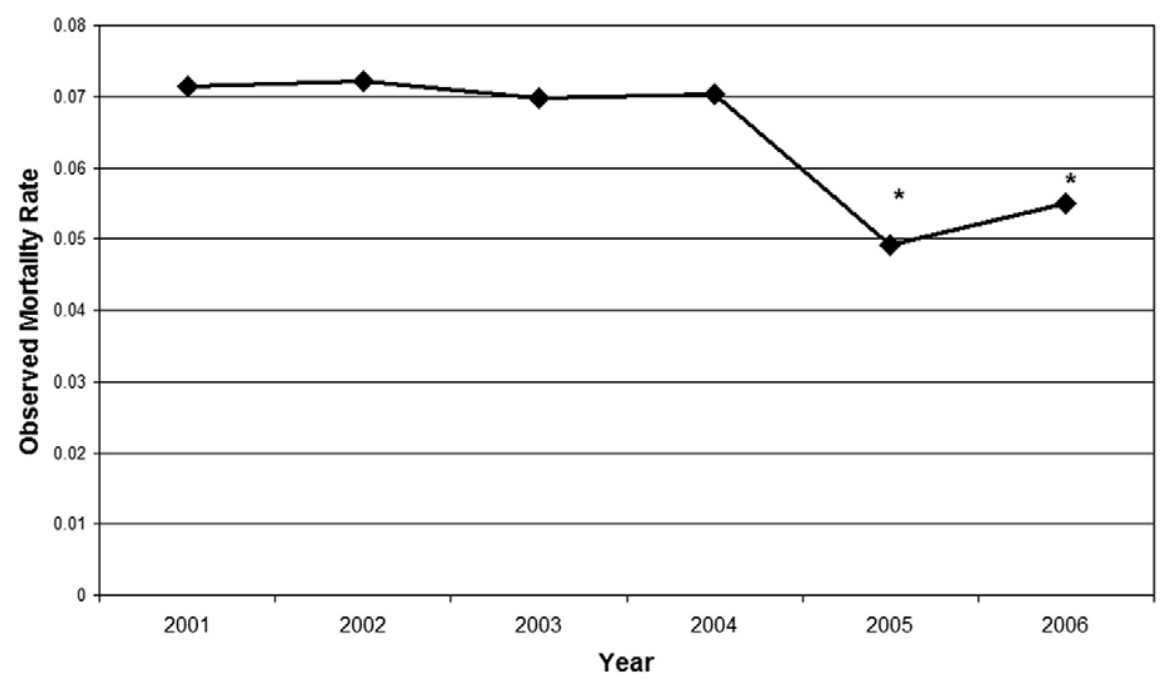

Figure 2. Surgical trauma ICU (STICU) mortality by year from 2001 to 2006 . The glucose control protocol was implemented in January 2005 , which was temporally associated with a decrease in the STICU mortality rate.

from 2003 to 2006 is displayed in Figure 1. In addition to progressively lower glucose levels, STICU glucose variability decreased over the study period. The mean observed mortality rates by year were: $2001,7.1 \% ; 2002,7.2 \%$; $2003,6.9 \% ; 2004,7.0 \% ; 2005,4.4 \%$; and 2006 year to date, $5.2 \%$. $(<\mathrm{p} 0.001$, period 1 versus period 2 and chi-square for trend). These mortality rates are depicted in Figure 2. The mean APACHE II acute physiology scores were 15.7 in $2003,15.6$ in 2004, and 15.4 in 2006 . Respective APACHE II-predicted mortality rates in 2003, 2004, and 2005 were $0.22,0.22$, and $0.21(\mathrm{p}=0.42)$. Using APACHE II methodology, the observed minus expected mortality rates were $-0.13(-59 \%),-0.15$ $(-68 \%)$, and $-0.17(-80 \%)$, respectively.

When comparing period 1 with period 2 , there was no difference with respect to pneumonia or bacteremia, but the incidence of intraabdominal abscess was less after implementation of the protocol $(2.1 \%$ versus $0.72 \%$, p $<$ 0.002 , Table 2).

\section{Trauma patients}

In the trauma patients, respective TRISS-predicted mortality rates in 2003, 2004, 2005, and 2006 were $0.23 \pm 0.01$, $0.20 \pm 0.01,0.20 \pm 0.01$, and $0.18 \pm 0.01$. The respective trauma observed mortality rates for 2003 to 2006 were $0.13,0.13,0.097$, and $0.11(\mathrm{p}=0.11$, period 1 versus period 2). The respective observed minus the expected mortality rates from 2003 to 2006 were $-0.10(-43 \%)$, $-0.07(-35 \%),-0.08(-40 \%)$, and $-0.07(-39 \%)$. Mean STICU lengths of stay comparing period 1 with period 2 (before and after protocol implementation) were 6.6 days \pm 0.26 versus 6.0 days $\pm 0.27(p=0.15)$. Mean hospital lengths of stay comparing the respective periods were $13.7 \pm 0.5$ days versus $12.9 \pm 0.5$ days $(p=0.26)$. Mean ventilator days comparing period 1 with period 2 were $3.1 \pm 0.3$ days versus 2.4 days \pm 0.2 days $(\mathrm{p}=0.03)$.

\section{DISCUSSION}

These data demonstrated that tight glucose control can be achieved through the use of a formal protocol, multidisciplinary commitment, careful monitoring, and implementation of a formal quality improvement nursing position in an ICU. The initiation of a formal protocol was associated with improved glucose control and a significant decrease in ICU mortality. In addition to better glucose control, the variation in glucose values was significantly reduced. Clinically significant hypoglycemia was rarely a problem in patients on the protocol, but hypoglycemia was more common after implementation of the protocol. The reduction in mortality did not appear to be related to a change in patient mix because severity of illness and injury remained constant over the study period. Subset analysis was limited by the total sample size and the retrospective nature of the study, but there was a reduction in total ventilator days in the trauma patients.

Table 2. Comparison of Infections Before and After Initiation of Glucose Control Protocol

\begin{tabular}{lccc}
\hline Infections & Period 1, \% & Period 2, \% & p Value \\
\hline Bacteremia & 8.9 & 7.8 & 0.28 \\
\hline Pneumonia & 3.5 & 3.3 & 0.98 \\
\hline Intraabdominal abscess & 2.1 & 0.7 & 0.002 \\
\hline
\end{tabular}

Pneumonia, bronchoalveolar lavage quantitative culture $\geq 10^{5}$ bacteria. 


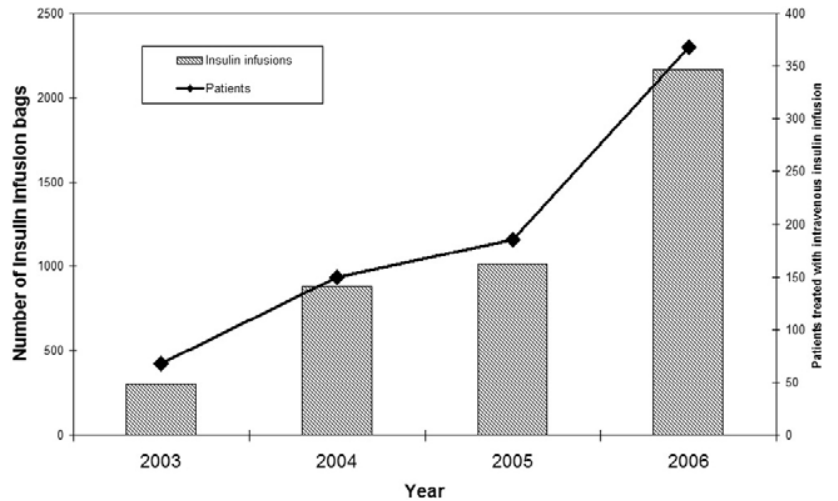

Figure 3. Intravenous insulin infusion use during the study period. Intravenous insulin use significantly increased during the study period.

\section{Limitations}

These data have the limitations inherent in a retrospective review. The greatest of these limitations may be that the improvement in glucose control cannot be causally tied to the reduction in mortality over the course of the study period. Several other protocols and care improvements in the unit occurred over the course of the study time period, so it is possible that the reduction in mortality was related to changes other than improved glucose control. Specifically, the process of developing the glucose control protocol was part of a collaborative effort between the nursing staff, the ICU faculty, the respiratory therapy staff, and the pharmacy staff to establish protocols and guidelines concerning common clinical problems in the STICU. The reduction in mortality may be a result of some other factor, or perhaps more likely, a combination of factors. The team knowledge and skills developed in the process of implementing the glucose control protocol could be potentially more beneficial than the glucose control itself.

Other potential confounding factors were a temporary loss of neurosurgery in 2003 and the arrival of a new surgical chairman (SMC) with a focus on surgical critical care. In summary, there was a temporally associated decline in mortality, but this change may have been from any one of a number of factors other than glucose control.

\section{Increased effort and cost}

Although we did not measure cost directly, there was almost certainly an increase in cost associated with tight glucose control in our STICU. The rise in cost resulted from the increased use of an insulin infusion, the increased nursing and physician time required to achieve euglycemia, and the increased number of bedside blood glucose values (Fig. 3). In the latter portion of the study period, we invested in a nursing quality improvement position. Although not exclusively dedicated to

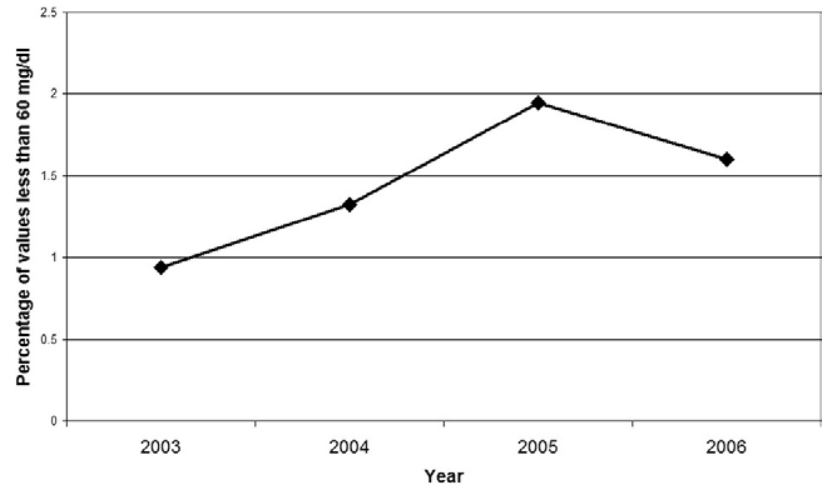

Figure 4. Percentage of point-of-care glucose tests with values of less than $60 \mathrm{mg} / \mathrm{dL}$. Although the absolute number of episodes was small, there was an increase in hypoglycemia during the study period.

glucose control, a portion of the cost of this salary is related to the process of tight glucose control.

Although these questions cannot be fully answered with our data, the increased costs are probably offset by the potential benefits of achieving euglycemia.

\section{Hypoglycemia}

In addition to the time and effort required to achieve tight glucose control, hypoglycemia is probably the biggest concern of most clinicians. Although not yet published, the Glucontrol trial, a multicenter, randomized, clinical trial examining tight glucose control as compared with a less strict regimen of glucose control, was stopped because of increased mortality in the small group of patients who developed hypoglycemia. From our data, clinically relevant hypoglycemic values on the bedside point-of-care glucose testing doubled from 2003 to 2005, and have remained significantly higher than they were in the baseline year of 2003 (Fig. 4). Although there was an increase in hypoglycemia, the absolute number of hypoglycemic patients was low. Because we examined the ratio of hypoglycemic to total blood glucose values, and patients who develop hypoglycemia are likely to have multiple repeat tests in followup to these low values, our data set probably overrepresents the actual number of patients with hypoglycemia. Nonetheless, hypoglycemia was more common after implementation of the regimen of tight glucose control. None of our patients had any evident neurologic sequelae from hypoglycemia (no seizures or persistent neurologic deterioration).

Hypoglycemia commonly occurred with some predictable and preventable patterns: continuation of insulin when enteral or parenteral feeding had been discontinued or suddenly held; insulin infusions in patients with progressive liver failure; and errors involving pump programming or unintended switching of infusion pumps, resulting in an unexpectedly high infusion rate. The reduction of 
hypoglycemia values in the last year of the study period probably reflects system improvements that reduced the frequency of these relatively predictable situations.

\section{Process improvement}

In our STICU, tight glucose control could not be achieved by relying on individual physicians and nurses. If our group of STICU faculty physicians had been surveyed in 2002 or 2003, we would have answered "yes" to two key questions: "Do you believe the weight-of-evidence supports efforts to achieve tight glucose control?" and, "Have you implemented measures to achieve tight glucose control in your own ICU?" Examination of our data, however, revealed that we clearly had not achieved tight glucose control. Only after implementing a formal protocol did we begin to achieve better glucose control, and only after establishment of a nursing monitor did we begin to see the majority of our patients within our target range of 80 to $110 \mathrm{mg} / \mathrm{dL}$.

The key steps in this process involved physician and nursing leadership; a multidisciplinary group responsible for development, and achievement of consensus, concerning the protocol; starting slow and thoroughly testing the protocol (initially one patient at a time); improved ability to monitor and track our bedside glucose levels; and routine monitoring of every patient in the STICU with reminders to implement the protocol on each patient with blood glucose values not within the target range.

Although tight glucose control is associated with increased effort and cost, our nurses and physicians are generally supportive and enthusiastic about implementation of the improvement. This emphasizes the importance of initial leadership, consensus, and the need to start slowly, and proceed steadily, while building on small successes.

\section{Subgroup analysis}

Subgroup analysis was limited because of the relatively small sample size and the retrospective nature of the majority of the data collection. But after implementation of the protocol, there appeared to be fewer intrabdominal abscesses in the total patient group and fewer days of mechanical ventilation in the trauma subset of patients. There were nonsignificant trends to fewer positive blood cultures and fewer trauma patient STICU days.

In conclusion, improvements in glucose control in the ICU can be achieved by using a protocol for intensive insulin therapy. In our STICU, this was temporally associated with a significant reduction in mortality, fewer intraabdominal abscesses, and fewer postinjury ventilator days. There was a small increase in the incidence of hypoglycemia. Our data support the notion that tight glucose control can be achieved by implementing a protocol and focusing on multidisciplinary intensive care unit performance improvement. Additional large scale, multicenter, randomized clinical trials will help determine whether the reduction in mortality is causally related and whether this strategy can be safely implemented by the majority of intensive care units.

\section{Author Contributions}

Study conception and design: Reed, Stewart, Sherman, Myers, Corneille, Larson, Gerhardt, Beadle, Gamboa, Dent, Cohn, Pruitt

Acquisition of data: Reed, Stewart, Sherman, Larson, Gerhardt, Beadle

Analysis and interpretation of data: Reed, Stewart, Myers, Cohn, Pruitt

Drafting of manuscript: Stewart

Critical revision: Reed, Stewart, Sherman, Myers, Corneille, Larson, Gerhardt, Beadle, Gamboa, Dent, Cohn, Pruitt

Acknowledgment: We gratefully acknowledge the support of the entire Surgical Trauma Intensive Care Unit staff for their help with the protocol and for their assistance in abstracting the charts Specifically, we thank Regina Reed, Tracy Wenzel, Denise Guerra, Daniel Rubalcaba, Ileana Fonseca, Susanne Thees, Regina Wilson, Sarah Kucera, Celina Gonzales, Debra Fraley, Flerida De Veas, Petra Ibarra, Reynaldo Ibarra, Michael Payne, Kimberly Ratliff, Pete Young, Brad Gilliam, Hope Rangel, Jason Proulx, and Jason Gourlas. We thank Marilyn McFarland, Lila Roebuck Canuelle, Thomas Golden, Lisa Wammack, Ellen Kirkman, Kristen Navarro, and Susan Spencer for their help with data, data analysis, and article preparation. We also thank the members of the University of Texas Intensive Care Unit Collaborative for their encouragement and their assistance in improving our glucose control protocol.

\section{REFERENCES}

1. Duane TM, Dechert T, Dalesio N, et al. Is blood sugar the next lactate? Am Surg 2006;72:613-617, discussion 617-618.

2. Laird AM, Miller PR, Kilgo PD, et al. Relationship of early hyperglycemia to mortality in trauma patients. J Trauma 2004; 56:1058-1062.

3. Yendamuri S, Fulda GJ, Tinkoff GH. Admission hyperglycemia as a prognostic indicator in trauma. J Trauma 2003;55:33-38.

4. Sung J, Bochicchio GV, Joshi M, et al. Admission hyperglycemia is predictive of outcome in critically ill trauma patients. J Trauma 2005;59:80-83.

5. Gore DC, Chinkes D, Heggers J, et al. Association of hyperglycemia with increased mortality after severe burn injury. J Trauma 2001;51:540-544. 
6. Vogelzang M, Nijboer JM, van der Horst IC, et al. Hyperglycemia has a stronger relation with outcome in trauma patients than in other critically ill patients. J Trauma 2006;60:873-877, discussion 878-879.

7. Gore DC, Wolf SE, Sanford AP, et al. Extremity hyperinsulinemia stimulates muscle protein synthesis in severely injured patients. Am J Physiol Endocrin Metabol 2004;286:E529-534.

8. Wu X, Thomas SJ, Herndon DN, et al. Insulin decreases hepatic acute phase protein levels in severely burned children. Surgery 2004;135:196-202.

9. Thomas SJ, Morimoto K, Herndon DN, et al. The effect of prolonged euglycemic hyperinsulinemia on lean body mass after severe burn. Surgery 2002;132:341-347.

10. Van den Berghe G, Wouters P, Weekers F, et al. Intensive insulin therapy in the critically ill patients.[see comment]. N Engl J Med 2001;345:1359-1367, 1368.

11. Van den Berghe G, Wilmer A, Hermans G, et al. Intensive insulin therapy in the medical ICU.[see comment]. N Engl J Med 2006;354:449-461, 462.

12. Wong DT, Barrow PM, Gomez M, McGuire GP. A comparison of the Acute Physiology and Chronic Health Evaluation (APACHE) II score and the Trauma-Injury Severity Score (TRISS) for outcome assessment in intensive care unit trauma patients. CritCare Med 1996;24:1642-1648.

13. Boyd CR, Tolson MA, Copes WS. Evaluating trauma care: the TRISS method: Trauma Score and the Injury Severity Score. J Trauma 1987;27:370-378.

\section{Discussion}

EDWARD E CORNWELL III, MD (Baltimore, MD): The neurohumoral response to critical injury and illness reflects a host of substances that promote tissue catabolism and mobilization of nutrient substrates. At the same time, the identification of elevated serum markers that identify insulin resistance, such as insulin-like growth factor binding protein, are correlated with increased mortality in critically ill patients. It is therefore not surprising that hyperglycemia has been identified as a surrogate risk factor for morbidity and mortality in critically ill and injured patients, and several clinical trials have evaluated the impact of intensive insulin therapy on outcomes. Although a well publicized, randomized control trial by Van Den Berghe and others in 2001 identified a survival benefit in critically ill patients receiving intensive therapy with a target glucose between 80 and $110 \mathrm{mg} / \mathrm{dL}$. Widespread implementation of such a protocol is hindered by incomplete identification of appropriate patient subsets and acceptable glucose values.

Imagine yourself as a pancreaticobiliary surgeon whose patient had a major liver resection or a Whipple procedure and is extubated, hemodynamically well perfused, and ready for ward transfer on post-op day two, or a trauma surgeon with a 24-year-old patient who is ready for ward transfer on post-injury day two following an abdominal gunshot wound; and the surgical intensivist tells you that the patient's transfer will be delayed by six hours or so as we wean the insulin drip, adjust the glucose infusion, or treat the hypoglycemia. It is precisely these areas needing further refinements for which Dr Stewart and colleagues are to be congratulated on their nicely presented work evaluating the im- plementation of an intensive insulin protocol. I appreciate their advanced submission of a well written manuscript in ample time for review.

The authors' findings that the implementation of the insulin protocol and overall improvement in glucose control was associated with an initial drop in mortality, needs more information before a cause and effect correlation can be evaluated. Specifically, the mean glucose levels and the mortality rates are presented for all patients admitted to the SICU during the study period rather than for those patients who were either eligible or actually received the insulin protocol. It is also not clear what proportion of SICU patients actually received the insulin protocol, and therefore the contribution this protocol makes to overall better glycemic control is undetermined.

The few years immediately preceding the initiation of your study saw significant activity in the surgical critical care literature leading to metaanalyses or evidence-based guidelines of evolving clinical protocols. Some of these protocols, such as early enteral feeds in head injured and other trauma patients, quantitative cultures leading to earlier antibiotic therapy, antifungal prophylaxis, and the aggressive screening for relative adrenal insufficiency leading to cortisol replacement therapy, would be expected to affect the random glucose levels you presented. Were any such protocols started or stopped during your study period?

Second, what was your definition of "hyperglycemia" for purposes of initiating the insulin infusion and when did you stop the infusion? The change of mean glucose values in your manuscript is slightly more modest, but all values presented were above the $110 \mathrm{mg}$ percent value in the Van Den Berghe paper that motivated this work.

Number three, what proportion of your study subjects were trauma patients? There is some suggestion in the literature, nicely covered by your references, that hyperglycemia carries a stronger correlation with outcomes in trauma patients than in others admitted to the SICU.

In summary, as we approach the glass ceiling of our abilities to resuscitate patients from critical injuries and surgical illnesses, we must further refine our focus on addressing the metabolic defects produced by the physiologic response to this stress. I applaud the authors for highlighting the challenges in addressing these defects.

MARTIN A CROCE, MD (Memphis, TN): The group from San Antonio has tried to shed some light on a very intriguing topic. First of all, I want to really commend Dr Stewart for his presentation, not just the nuts and bolts of the presentation, but he takes a very difficult protocol outlining the true hazards and the real life problem with implementing said protocol and honestly evaluates the complications associated with implementing said protocol. And with that being said, this entire concept of hyperglycemia is associated with increased infectious morbidity is really not very difficult to believe.

After the initial excitement about tight glucose control is leveled off a bit, Dr Stewart and his colleagues have tried to shed some light on this topic. It demonstrates decreased abdominal abscesses and mortality that was coincident with their institution of this tight glucose control protocol. Personally, I remain somewhat skeptical of this entire concept and hope that Dr Stewart will be able to convince me that basic normalization of glucose in critically ill patients is really a good idea.

My first question. Nineteen percent of your patient population was diabetic upon admission to the hospital. What were the out- 